\title{
ASSOCIATION BETWEEN MATERNAL SERUM VITAMIN D AND EARLY PREGNANCY SPONTANEOUS ABORTION IN IRAQI WOMEN
}

\author{
ASHWAQ KADHIM MOHAMMED*, VIAN HUSSAM ALMANSI ALQANI
}

Department of Obstetrics and Gynecology, College of Medicine, University of Al-Qadisiyah, Iraq. Email: ashwaqalshemeri@gmail.com Received: 25 December 2017, Revised and Accepted: 06 January 2018

\section{ABSTRACT}

Background: Spontaneous pregnancy loss is seen in approximately 10-15\% of clinically observed pregnancies.

Objective: This study was aimed to estimate the Vitamin D serum concentration in a cohort of women with a history of recurrent spontaneous abortion.

Methods: The current cross-sectional study was conducted at Al-Diwaniya maternity and child teaching hospital, Al-Diwaniya province, Iraq. Data collection, lab work, and procedures related to the study extended from August 2016 to August 2017. The study enrolled 42 women of childbearing age who have a history of spontaneous early pregnancy loss. From each woman, a venous sample was obtained and sent to a lab to assess serum Vitamin D concentrations using standard routine biochemical methods.

Results: Assessment of mean maternal serum Vitamin D concentration revealed that the mean was $21.48 \pm 11.82$ (ng/ml) and the range was from 5 to 50 (ng/ml). The number of women with low serum Vitamin D ( $<20 \mathrm{ng} / \mathrm{ml}$ ) was 25 (approximately $60 \%)$, the results also revealed a highly negative correlation was obtained between number of abortions and maternal serum Vitamin $\mathrm{D}(\mathrm{r}=-0.717, \mathrm{p}<0.001)$ and that the value or $\mathrm{R}^{2}$ was 0.514 which indicated that the level of Vitamin D alone can act as an independent predictor to explain 51.4\% of spontaneous abortions in the participating women.

Conclusion: Vitamin D level is a strong predictor of pregnancy loss in early pregnancy, and that correction of Vitamin D status among Iraqi pregnant ladies may substantially reduce the frequency of spontaneous abortion.

Keywords: Early pregnancy abortion, Vitamin D deficiency, Serum vitamin.

(C) 2018 The Authors. Published by Innovare Academic Sciences Pvt Ltd. This is an open access article under the CC BY license (http://creativecommons. org/licenses/by/4. 0/) DOI: http://dx.doi.org/10.22159/ajpcr.2018.v11i2.24588

\section{INTRODUCTION}

One of the major pregnancy adverse outcomes is baby loss before completion of natural pregnancy period, and when conception does not end with a live born child this is termed pregnancy loss; when it happens before 28 weeks gestation, it is called abortion [1]. Spontaneous pregnancy loss is seen in approximately $10-15 \%$ of clinically observed pregnancies. Early pregnancy loss (spontaneous abortion) is assigned when the loss occurs before completion of 12 weeks gestation [2] because the majority of spontaneous pregnancy loss happens early (before 12 weeks gestation), a thorough study of the pathophysiology behind this adverse pregnancy outcome is of great importance. A lot of mechanisms have been proposed with this regard, including hormonal derangements, chromosomal anomalies, intrauterine infections, uterine abnormalities, thrombophilia, and autoimmune disorders; in approximately $50 \%$ of cases with early pregnancy loss, the causes remain enigmatic in which the loss is termed unexplained pregnancy loss [3].

Vitamin D3 (1,25 dihydroxy Vitamin D3) is a hormone that is water soluble and well known to function in promoting bone mineralization and maintaining calcium homeostasis [4]. Moreover, it-D3 plays a major role in contorting cell proliferation and maturation and in modulating immune response both innate and adaptive form $[5,6]$.

A lot of epidemiological published literature refers to the global occurrence of Vitamin D deficiency and insufficiency. Mitchell et al. [7] recorded the Vitamin D deficiency incidence (a serum level of $<20 \mathrm{ng} / \mathrm{mL}$ ) in women during reproductive age to be $31 \%$. Reports from the "National Health and Nutrition Examination Surveys" described that the problem is variable between racial and ethnic categories, "with nonHispanic blacks possessing a higher rate of Vitamin D deficiency and" insufficiency when compared to white ethnic groups [8] these results were supported "by Forrest and Stuhldreher, who registered" that blacks had the highest incidence of Vitamin D insufficiency (82.1\%) and then followed by Hispanics (69.2\%) [9]. In spite of its global prevalence, wide geographical screening for vitamin insufficiency has not been adopted because of the expensive serum assays. For that reason, screening has been carried out only for groups at great risk for Vitamin Deficiency [10].

Recently, a great deal of attention has been focused on vitamin status during pregnancy. Evidence about the role of Vitamin D in human reproduction has been proved in a number of researchers [11]. Globally, vitamin deficiency is a frequent problem among women at the age of childbearing $[12,13]$ among obstetric complications that have been reported to be associated with Vitamin Deficiency are gestational diabetes [14], pre-eclampsia [15] impaired intrauterine growth [16], and bacterial vaginosis [17]. Despite that, the association between poor Vitamin D status and spontaneous early pregnancy loss is less well defined.

A number of studies showed an association between pregnancy loss and vitamin deficiency that is probably mediated by "effector CD4+ T helper (Th) cellular responses in the innate (Th1) and adaptive (Th2) immune systems." Vitamin D is proposed to promote the Th2 effect by increasing interleukin (IL-5), (IL)-4, and IL-13 and preventing the Th1 response of IL-2, IL-1, tumor necrosis factor- $\alpha$ (TNF- $\alpha$ ), and interferon- $\gamma$ (IFN- $\gamma$ ) [18]. "In a study including 133 women with recurrent pregnancy loss, Ota et al. showed that 63 out of 133 women (47.4\%) had Vitamin D deficiency and a significant association with natural killer cell activity with Vitamin D deficiency" [19]. Moreover, in another study of 115 female patients with antiphospholipid syndrome 
(APS), Vitamin D concentrations in APS women were significantly less as compared to healthy control group [20]. Tavakoli et al. observed same cytokine profiles and Vitamin D expression in endometrial cells taken from female patients having unexplained recurrent pregnancy loss as compared to healthy control group [21]. Hence, the aim of the present study was to estimate the Vitamin D serum concentration in a cohort of women with a history of recurrent spontaneous abortion.

\section{METHODS}

The current cross-sectional study was conducted at Al-Diwaniya maternity and child teaching hospital, Al-Diwaniya province, Iraq. Data collection, lab work, and procedures related to the study extended from August 2016 to August 2017. The study enrolled 42 women of childbearing age who have a history of spontaneous early pregnancy loss. From each woman, a venous sample was obtained and sent to a lab to assess serum Vitamin D concentrations using standard routine biochemical methods.

\section{Statistical analysis}

Data were transformed into a spreadsheet of SPSS version 23 . Numeric variables were expressed as mean and standard deviation whereas nominal data were expressed as number and percentage. Correlation between serum Vitamin D level and a number of abortions was evaluated using Spearman's test. The level of significance was considered at $\mathrm{p} \leq 0.05$.

\section{RESULTS}

Then, the present study included 42 women at reproductive age and had a history of spontaneous abortions. The age was ranging from 17 to 36 years, and the mean age was $24.95 \pm 5.09$ years. A number of spontaneous abortions were ranging from 1 to 6 and the mean was. Assessment of mean maternal serum Vitamin D concentration revealed that the mean was $21.48 \pm 11.82(\mathrm{ng} / \mathrm{ml})$ and the range was from 5 to $50(\mathrm{ng} / \mathrm{ml})$. The number of women with low serum Vitamin $D(<20 \mathrm{ng} / \mathrm{ml})$ was 25 (approximately 60\%), as shown in Table 1.

There was no significant correlation between maternal age and serum Vitamin D concentrations ( $r=0.091, p=0.568$ ), in other words, low level of the vitamin was evenly distributed along the scale of age and that no reproductive age is immune from having Vitamin D deficiency, as shown in Fig. 2. Nevertheless, highly negative correlation was obtained between number of abortions and maternal serum Vitamin $D(r=-0.717, p<0.001)$ and that the value or $\mathrm{R}^{2}$ was 0.514 which indicated that the level of Vitamin D alone can act as an independent predictor to explain $51.4 \%$ of spontaneous abortions in the participating women, as shown in Fig. 2.

\section{DISCUSSION}

The current study showed a relatively great rate of vitamin deficiency among women with history with spontaneous early pregnancy miscarriage which was approximately $60 \%$. Similar results were obtained by several other investigators [12,13]. "In a study including 133 women with recurrent pregnancy loss, Ota et al. showed that 63 out of 133 women (47.4\%) had Vitamin D deficiency [19]." Moreover, a significant correlation was found between a number of abortions and serum concentration of Vitamin D.

Vitamin D status is poorly categorized as an obstetric problem in Iraqi women and little if any study has linked spontaneous abortion to recurrent spontaneous early pregnancy abortion. The cause of

Table 1: Characteristics of the study populations

\begin{tabular}{lll}
\hline Characteristic & Mean \pm SD & Range (min-max) \\
\hline Age (years) & $24.95 \pm 5.09$ & $19(17-36)$ \\
Number of abortion & $2.57 \pm 1.42$ & $6(1-6)$ \\
Vitamin D (ng/ml) & $21.48 \pm 11.82$ & $45(5-50)$ \\
\hline
\end{tabular}

SD: Standard deviation

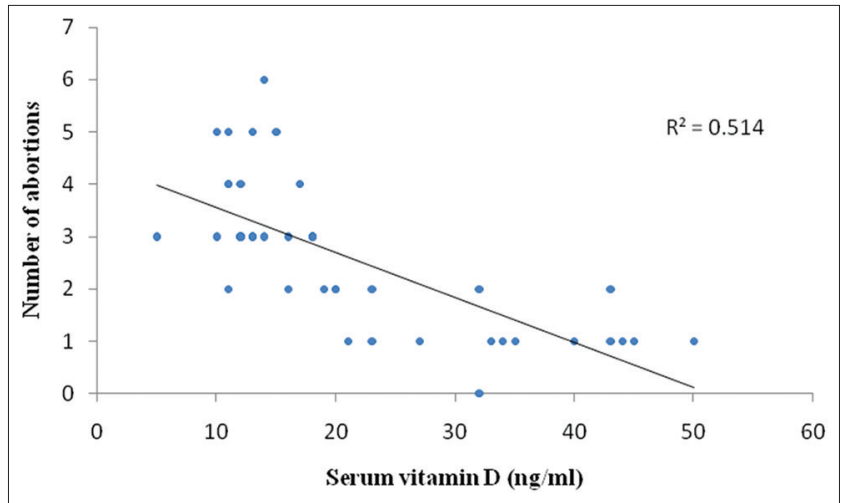

Fig. 1: Correlation between maternal serum Vitamin D concentration and number of spontaneous abortions

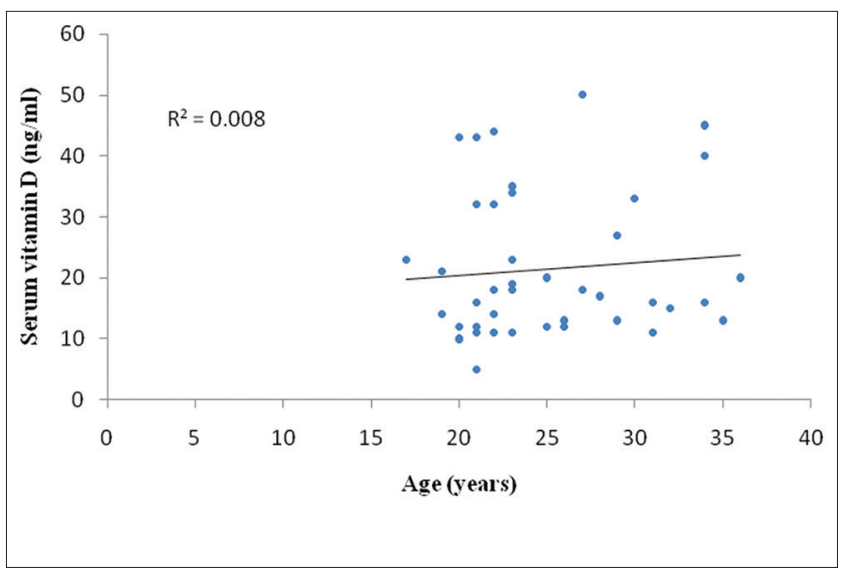

Fig. 2: Correlation between maternal age and serum Vitamin D concentration

pregnancy loss in women with insufficient Vitamin D levels has been investigated by several authors. It is probably mediated by "effector CD4+ Thelper (Th) cellular responses in the innate (Th1) and adaptive (Th2) immune systems." Vitamin is proposed to promote them Th2 effect by increasing IL-5, (IL)-4, and-13 and preventing then Th1 response of IL-2, IL-1, TNF- $\alpha$, and IFN- $\gamma[18]$.

\section{CONCLUSION}

Vitamin D level is a strong predictor of pregnancy loss in early pregnancy, and that correction of Vitamin D status among Iraqi pregnant ladies may substantially reduce the frequency of spontaneous abortion.

\section{REFERENCES}

1. Hou W, Yan XT, Bai CM, Zhang XW, Hui LY, Yu XW, et al. Decreased serum vitamin D levels in early spontaneous pregnancy loss. Eur J Clin Nutr 2016;70:1004-8.

2. Yinping H. Spontaneous abortion. In: Yue J, editor. Obstetrics and Gynecology. $7^{\text {th }}$ ed. Beijing: People's Health Publishing House; 2008. p. 83-6.

3. Practice Committee of the American Society for Reproductive Medicine. Evaluation and treatment of recurrent pregnancy loss: A committee opinion. Fertil Steril 2012;98:1103-11.

4. Holick MF. Vitamin D deficiency. N Engl J Med 2007;357:266-81.

5. Bikle DD. Vitamin D regulation of immune function. Vitam Horm 2011;86:1-21.

6. Lagishetty V, Liu NQ, Hewison M. Vitamin D metabolism and innate immunity. Mol Cell Endocrinol 2011;347:97-105.

7. Mitchell DM, Henao MP, Finkelstein JS, Burnett-Bowie SA. Prevalence and predictors of vitamin D deficiency in healthy adults. Endocr Pract 2012;18:914-23. 
8. Looker AC, Johnson CL, Lacher DA, Pfeiffer CM, Schleicher RL, Sempos CT. Vitamin D status: United States, 2001-2006. NCHS Data Brief 2011;59:1-8.

9. Forrest KY, Stuhldreher WL. Prevalence and correlates of vitamin D deficiency in US adults. Nutr Res 2011;31:48-54.

10. Kennel KA, Drake MT, Hurley DL. Vitamin D deficiency in adults: When to test and how to treat. Mayo Clin Proc 2010;85:752-7.

11. Lerchbaum E, Rabe T. Vitamin D and female fertility. Curr Opin Obstet Gynecol 2014;26:145-50.

12. Woo J, Lam CW, Leung J, Lau WY, Lau E, Ling X, et al. Very high rates of vitamin $\mathrm{D}$ insufficiency in women of child-bearing age living in Beijing and Hong Kong. Br J Nutr 2008;99:1330-4.

13. Song SJ, Zhou L, Si S, Liu J, Zhou J, Feng K, et al. The high prevalence of vitamin D deficiency and its related maternal factors in pregnant women in Beijing. PLoS One 2013;8:e85081.

14. Cho GJ, Hong SC, Oh MJ, Kim HJ. Vitamin D deficiency in gestational diabetes mellitus and the role of the placenta. Am J Obstet Gynecol 2013;209:560.

15. Bodnar LM, Catov JM, Simhan HN, Holick MF, Powers RW, Roberts JM, et al. Maternal vitamin D deficiency increases the risk of preeclampsia. J Clin Endocrinol Metab 2007;92:3517-22.
16. Leffelaar ER, Vrijkotte TG, van Eijsden M. Maternal early pregnancy vitamin D status in relation to fetal and neonatal growth: Results of the multiethnic Amsterdam born children and their development cohort. $\mathrm{Br}$ J Nutr 2010;104:108-17.

17. Bodnar LM, Krohn MA, Simhan HN. Maternal vitamin D deficiency is associated with bacterial vaginosis in the first trimester of pregnancy. J Nutr 2009;139:1157-61.

18. Dakhil, AS. Association of serum concentrations of proinflammatory cytokines and hematological parameters in rheumatoid arthritis patients. J Pharm Sci Res 2017;9:1966-74.

19. Ota K, Dambaeva S, Han AR, Beaman K, Gilman-Sachs A, KwakKim J, et al. Vitamin D deficiency may be a risk factor for recurrent pregnancy losses by increasing cellular immunity and autoimmunity. Hum Reprod 2014;29:208-19.

20. Andreoli L, Piantoni S, Dall'Ara F, Allegri F, Meroni PL, Tincani A. Vitamin D and ant phospholipid syndrome. Lupus 2012;21:736-40.

21. Tavakoli M, Jeddi-Tehrani M, Salek-Moghaddam A, Rajaei S, Mohammadzadeh A, Sheikhhasani S, et al. Effects of $1,25(\mathrm{OH}) 2$ vitamin D3 on cytokine production by endometrial cells of women with recurrent spontaneous abortion. Fertil Steril 2011;96:751-7. 